\title{
Using Evolution Strategies to Perform Stellar Population Synthesis for Galaxy Spectra from SDSS
}

\author{
J. C. Gomez and O. Fuentes
}

\begin{abstract}
Current surveys from modern astronomical observatories contain a huge amount of data; in particular, the Sloan Digital Sky Survey (SDSS) has reached the order of terabytes of data in images and spectra. Such an amount of information needs to be exploited by sophisticated algorithms that automatically analyze the data in order to extract useful knowledge from the mega databases.

In this work we employ Evolution Strategies (ES) to automatically extract a set of physical parameters corresponding to stellar population synthesis (ages, metallicities, reddening and relative contributions) from a sample of galaxy spectra taken from SDSS. Such parameters are useful in cosmological studies and for understanding galaxy formation, composition, and evolution.

We pose this parameter extraction as an optimization problem and then solve it using ES. The idea is to reconstruct each galaxy spectrum from the sample by means of a linear combination of three similar theoretical models, each contributing in a different way to the stellar population synthesis. This linear combination produces a model spectrum that is compared with the original spectrum using a simple difference function. The goal is to find a model that minimizes this difference, using ES as the algorithm to explore the parameter space.

We present experimental results using a set of 100 spectra from SDSS Data Release 2 that show that ES are very well suited to extract stellar population parameters from galaxy spectra.
\end{abstract}

\section{INTRODUCTION}

Evolution Strategies (ES) [1] are a very flexible and effective optimization algorithm [2], suitable to be employed in many areas and subjects such as mechanical design [3], optics [4] and control engineering [5]. In this paper we introduce a novel application of this method to a very interesting problem in astronomy: the fitting of galaxy spectra with models of stellar population synthesis; a problem that has been attacked following traditional approaches like $\chi^{2}$ minimization [6] or stochastic methods such as Simulated Annealing [7]. However, these methods have problems because they tend to be computationally expensive [7].

Astronomy faces today an important set of difficult tasks that need to be tackled by sophisticated algorithms. Basically there are two types of problems in modern computational astronomy: problems with great computational complexity (e.g. n-body and cosmological simulations) [8] and problems where a huge amount of information must be analyzed (e.g. sky surveys). The problem presented here belongs to the second category.

J. C. Gomez is with the Department of Computer Science, INAOE, Tonantzinla, Puebla, Mexico(phone: 52-222-2663100; fax: 52-222-2663152; email: jcgc@inaoep.mx).

O. Fuentes is with the Department of Computer Science,UTEP, El Paso, Texas, USA (phone: 915-7476956; email: ofuentes@utep.edu).
These tasks, where a huge amount of data is available to be analyzed, need to be performed automatically using reliable and efficient algorithms, because doing them manually is practically impossible. In particular, Sloan Digital Sky Survey (SDSS) [7] contains terabytes of information from galaxy images and spectra. This enormous amount of highquality data will be one of the central parts for gaining an enormous progress in our understanding of galaxies and the universe in general.

Galaxy spectra encode information about age and metallicity distributions of the constituent stars, which in turn reflect the star formation and chemical histories of the galaxies [9]. Extracting such information from observational data in a reliable way is crucial for a deeper understanding of galaxy formation, composition, and evolution.

In order to extract knowledge from SDSS spectra, a methodology must be set up to go from the observed spectra to physical properties of galaxies, including reddening, age, metallicity, and contribution of each stellar population. The method presented here is based on using ES for fitting, efficiently and automatically, an observed spectrum with a linear combination of simple theoretical stellar populations computed with evolutionary synthesis models at the same spectral resolution as that of the SDSS, trying to find the model that best matches the original spectra.

In this paper we have obtained results for a set of 100 spectra from SDSS Data Release 2 as a first approach to the use of ES in this problem, proving ES are very well suited to extract stellar population parameters from galaxy spectra automatically and efficiently.

The rest of the paper is structured as follows: Section 2 presents a brief description of galaxy spectra and the information we can extract from them, also in this section the data from SDSS is described, Section 3 contains the details of the implementation of ES to solve the problem, in Section 4 results are presented and Section 5 shows the conclusions and the future work.

\section{Galaxy Spectra}

The light of a star can be dispersed into a spectrum by means of a prism or a diffraction grating. The stellar spectrum is the distribution of the energy flux density over frequency. A stellar spectrum is made up by a continuum and by absorption and emission lines, which are produced by the different chemical elements present in the star [9]. Each line is a fingerprint of some chemical element that is excited or ionized. 
Almost all of the relevant information about physical properties of stars can be obtained more or less directly from studies of their spectra. The line shapes contain detailed information about chemical elements and atmospheric processes.

The spectrum of a galaxy, in fact, is the combination of spectra from the millions of stars in the galaxy. Thus the colors in a galaxy depend both on the ages of the stars (young stars are bluer) and on the abundance of heavy elements $Z$, or metallicity (stars with larger $Z$ are redder). The interpretation of the observational results thus has to be based on detailed modeling of the stellar composition of galaxies, or stellar population synthesis, where a several models of stellar populations are combined to reproduce galaxy spectra.

Studying the features of a galaxy spectrum tells us about the types of stars the galaxy contains, and the relative abundances of each type of star. Stars of different spectral classes contribute different characteristic absorption features to the galaxy spectrum. By observing the strength of various spectral features, we can find out about masses, ages, and chemical compositions of the stars that form the galaxy.

In galaxies we can distinguish three types of stellar populations: Type I that consists of young stars (hundreds of millions of years of age) with high metallicity, Type II that is composed by old stars almost without metallicity and Type III, an intermediate population with ages between Types I and II. There exist libraries of models of entire stellar systems for each type of population, with different ages and metallicities. Combinations of models of these three types of populations are used in stellar population synthesis to reproduce galaxy spectra.

In this work we employ a library of 50 models from Bressan et al. [10]. These models cover the following ages: $\quad$ young $=\left[10^{6}, 10^{6.3}, 10^{6.6}, 10^{7}, 10^{7.3}\right]$ years, intermediate $=\left[10^{7.6}, 10^{8}, 10^{8.3}\right] \quad$ years, old $=\left[10^{9}, 10^{10.2}\right]$ years and the following metallicities: $[0.0004,0.004,0.008,0.02,0.05] Z_{\odot}$ (solar metallicity).

An example of how to model a galaxy spectrum is shown in Figure 1, where three models of stellar population with different ages and metallicities are combined to create a simulated galaxy spectrum (thick line).

Thus, in order to extrapolate from observed spectra to physical properties of galaxies, it is necessary to find the best combination of models that fits the observed spectra. Performing this task is not easy, due to the number of parameters and the noise inherent to observational data, thus an effective and efficient algorithm must be employed to examine the space of potential solutions.

\section{A. SDSS Data}

SDSS is one of the most ambitious projects performed to survey the sky, trying to cover one quarter of the sky with high detail. To do this, the SDSS team has used a 2.5 meter telescope located in the observatory in Apache Point, New Mexico. This survey includes photometric and spectroscopic data.
TABLE I

DOMAINS FOR STELLAR POPULATION PARAMETERS

\begin{tabular}{|l|l|c|}
\hline \hline & Name & Domain \\
\hline \hline$a_{1}$ & young age & $\{1,2,3,4,5\}$ \\
$a_{2}$ & intermediate age & $\{6,7,8\}$ \\
$a_{3}$ & old age & $\{9,10\}$ \\
$m_{1}$ & metallicity population I & $\{1,2,3,4,5\}$ \\
$m_{2}$ & metallicity population III & $\{1,2,3,4,5\}$ \\
$m_{3}$ & metallicity population II & $\{1,2,3,4,5\}$ \\
$r_{1}$ & reddening population I & {$[-1,1]$} \\
$r_{2}$ & reddening population III & {$[-1,1]$} \\
$r_{3}$ & reddening population II & {$[-1,1]$} \\
$c_{1}$ & contribution population I & {$[0,1]$} \\
$c_{2}$ & contribution population III & {$[0,1]$} \\
$c_{3}$ & contribution population II & {$[0,1]$} \\
\hline \hline
\end{tabular}

Spectrography in SDSS is done using 2 spectrographs, covering a range from $3800 \AA$ to $9000 \AA$, with a resolution of $R=\lambda / \Delta \lambda=1800$. The Data Release (DR) 2 from SDSS [11], contains the spectra from 574 plates with 640 fibers each, covering an area of 2627 square degrees, with a total of 367,360 spectra of galaxies, quasars and stars. In Figure 2 we observe an example of a galaxy spectrum from SDSS DR2.

In this work we employ a sample of 100 spectra taken from SDSS DR2 that were used and analyzed in [12].

All the spectra from the sample are passed first to a preprocessing module where they were brought to the rest frame, using the redshift given in the SDSS database, corrected for extinction, using the maps given by Schlegel, Finkbeiner \& Davis [13] and the extinction law of Cardelli [14], sampled from $3800 \AA$ to $8000 \AA$ in steps of $1 \AA$ and normalized by the median flux in the $4010 \AA-4060 \AA$. All this process is done to simplify the fitting task and it is necessary to standardize all the data (the observed and the models).

\section{FITTING}

In order to fit an observed spectrum, it is necessary to define a mathematical model that, using the library of stellar population spectra, can produce simulated galaxy spectrum that matches the observation.

From the spectra library we can define ages and metallicities for each stellar population; nevertheless, to produce more realistic galaxy spectra we also need to know the reddening term and the relative contribution for each stellar population.

Thus, a model for a galaxy spectrum is constructed using the equation:

$$
\vec{g}=\sum_{i=1}^{3} c_{i} s_{i}\left(a_{i}, m_{i}\right)\left(10^{-4 r_{i} k}\right)
$$

where $s_{i}\left(a_{i}, m_{i}\right)$ represents a model of a stellar population from a library for specific age $\left(a_{i}\right)$ and specific metallicity $\left(m_{i}\right), 10^{-4 r_{i} k}$ is the reddening term, and $c_{i}$ is the relative contribution of each population.

Then, the final goal in this fitting task is to find, efficiently and automatically, the best combination for the following 


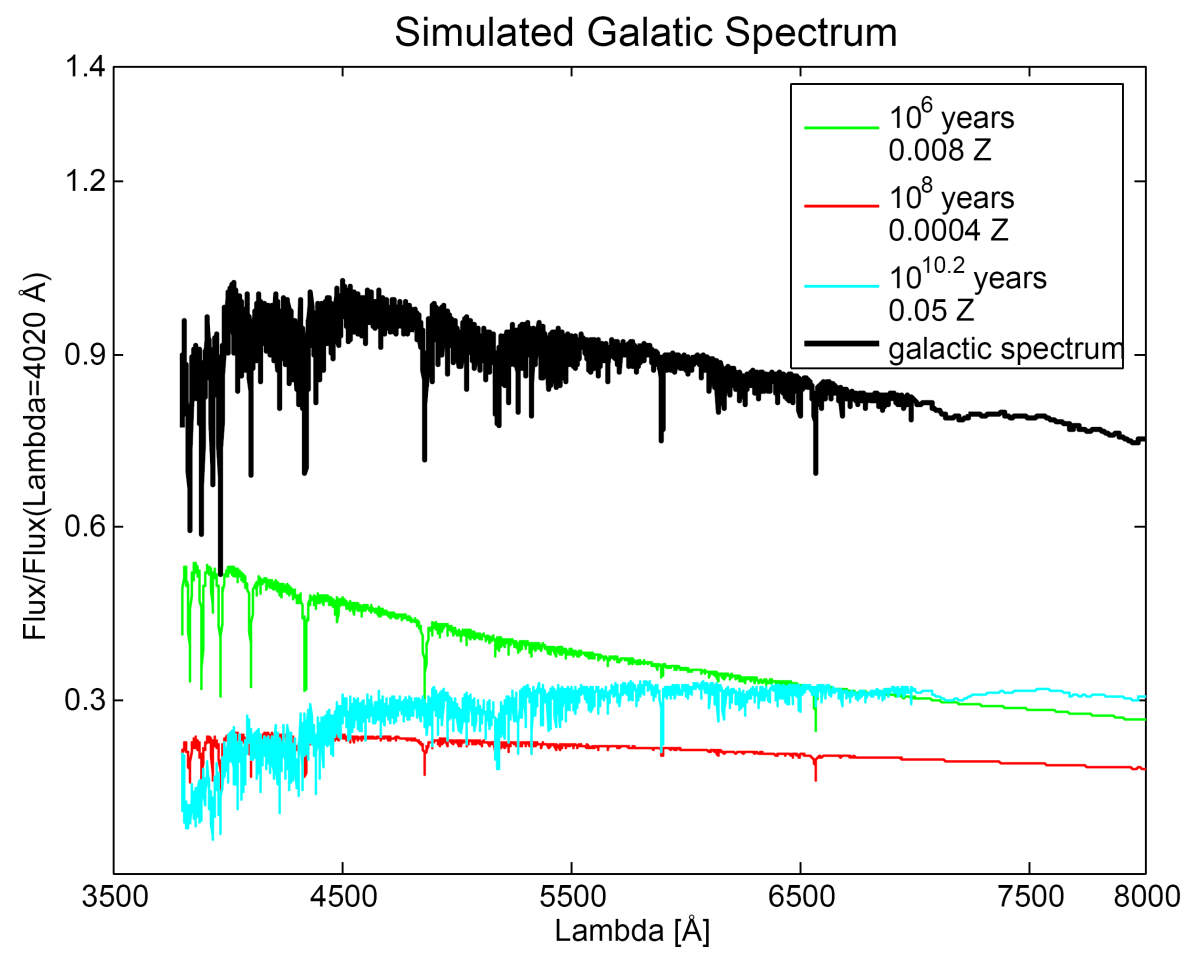

Fig. 1. Example of a simulated galaxy spectrum using three models of stellar population with different ages and metallicities. Spectrum is normalized in the region $\lambda=4020 \stackrel{\AA}{\circ}$

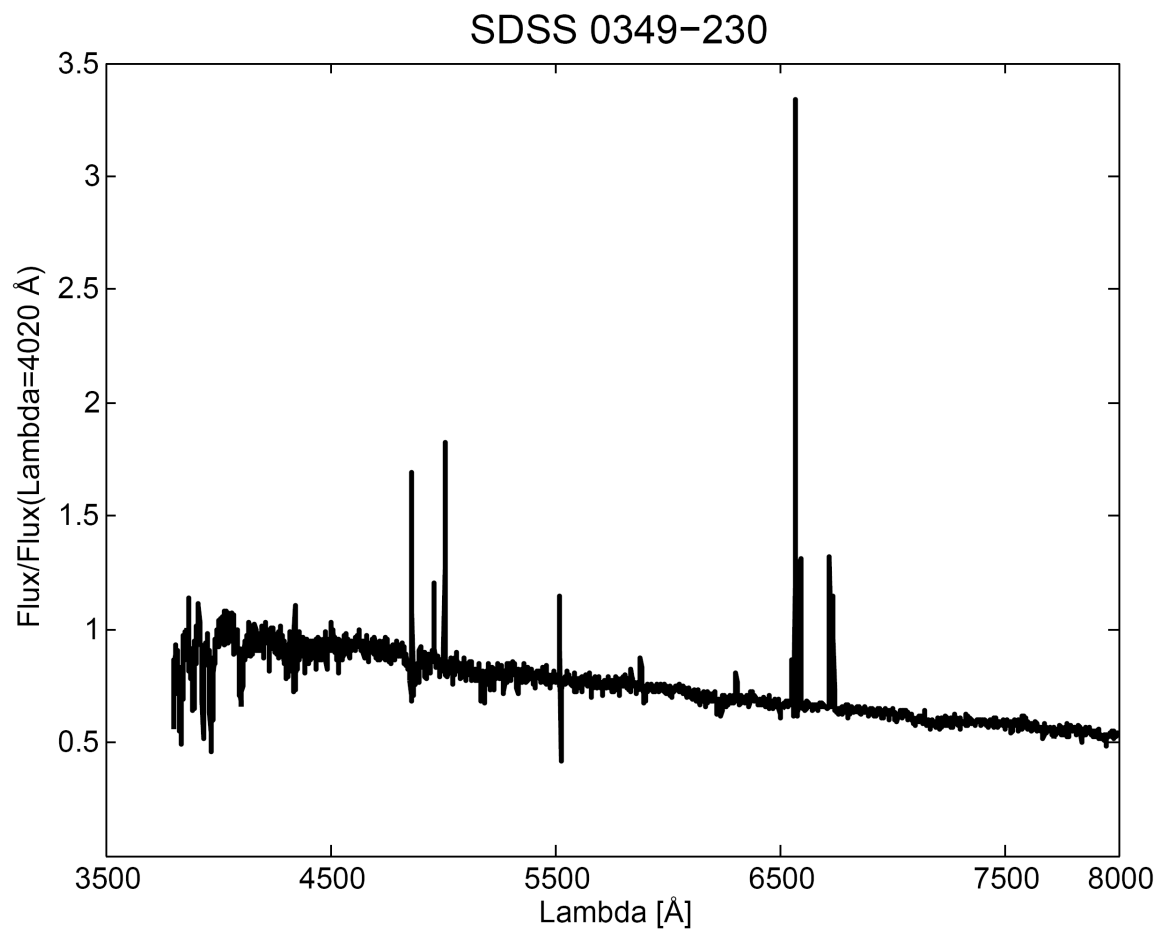

Fig. 2. Example of a galaxy spectrum from SDSS. Spectrum is normalized in the region $\lambda=4020 \AA$ 
stellar population parameters: reddening $\left(r_{1}, r_{2}, r_{3}\right)$, metallicities $\left(m_{1}, m_{2}, m_{3}\right)$, ages $\left(a_{1}, a_{2}, a_{3}\right)$ and relative contributions $\left(c_{1}, c_{2}, c_{3}\right)$ for stellar populations, that produce a model that matches the observed spectrum.

The stellar population parameters are shown in Table I together with the domains where they can fall. The parameters for age and metallicity are discrete, since they are indexes in the simulated stellar population spectra library, while reddening and contribution are real.

The models of stellar populations from the library are defined only for the stellar component of galaxies, which means absorption lines. Emission lines come from gas and dust in galaxies, so, since this component is not considered by the models, we need to discard the parts where emission lines are present in observed spectrum. Even with this restriction, the models from the library are adequate to describe the main features of the spectra.

There are some well know emission lines that are commonly present in galaxy spectra, such lines correspond with some chemical elements, and some of them can be observed in Figure 1. We have created a mask with the points where such emission lines are present to determine the 0 values for $w_{\lambda}$. The points masked are: $\left[\mathrm{Ne}_{\mathrm{III}}\right] \lambda=[3865, \ldots, 3873], \mathrm{H}_{\epsilon}, \mathrm{H}_{\delta}, \mathrm{H}_{\gamma}, \mathrm{H}_{\beta}$, $\left[\mathrm{O}_{\mathrm{III}}\right] \lambda=[3865, \ldots, 3873],\left[\mathrm{He}_{\mathrm{I}}\right] \lambda=[5871, \ldots$,

$5881], \mathrm{NaD} \lambda=[5885, \ldots, 5895],\left[\mathrm{O}_{\mathrm{I}}\right] \lambda=[6295, \ldots, 6305]$, $\left[\mathrm{N}_{\mathrm{II}}\right], \mathrm{H}_{\alpha} \lambda=[6543, \ldots, 6588]$ and $\left[\mathrm{S}_{\mathrm{II}}\right] \lambda=$ $[6712, \ldots, 6736]$.

\section{A. Stellar Population Synthesis as an Optimization Process}

We have observed that the present fitting task can be posed as an optimization problem in the following way: let $\vec{o}$ be the observed galaxy spectrum variable, let $\vec{g}$ be the simulated spectrum with the same dimensionality as $\vec{o}$. In order to measure how well a model $\vec{g}$ describes the spectrum $\vec{o}$ we use the following function:

$$
f(\vec{g})=\sum_{\lambda=3800}^{\lambda=8000}\left|o_{\lambda}-g_{\lambda}\right| w_{\lambda}
$$

where each $w_{\lambda}$ takes a value of 0 for emission lines and 1 otherwise. This is the fitness function used by our evolutionary algorithm. Thus, the goal of the optimization process is to obtain a model $\overrightarrow{g *}$ that minimizes the previous function, with $f(\vec{g} *)=0$.

Our implementation of ES for the present problem of fitting galaxy spectra with stellar population models is as follows: the initial population of ES $\vec{x}_{i} i=1, \ldots, \mu$ where $\vec{x}_{i}=$ $\left[a_{1, i}, a_{2, i}, a_{3, i}, m_{1, i}, m_{2, i}, m_{3, i}, r_{1, i}, r_{2, i}, r_{3, i}, c_{1, i}, c_{2, i}, c_{3, i}\right]$ and their corresponding strategy parameters vectors $\vec{\sigma}_{i}$ $i=1, \ldots, \mu$ are formed by randomly generated values for each parameter inside the domains defined in Table I. Then, each $\vec{x}_{i}$ is passed to a module that combines the stellar population models from the library following equation 1 to create the simulated galaxy spectrum $\vec{g}_{i}$. Afterwards, each $\vec{g}_{i}$ is evaluated using the fitness function.
The next step consists of an iterative process where if some model has a good match with the real spectrum we stop the process, otherwise we create $\lambda$ new individuals $\vec{x}_{j}^{\prime}$ $j=1, \ldots, \lambda$ using recombination, mutation and average operators (described in detail below). Then we create their corresponding models $\vec{g}_{j}^{\prime}$ and evaluate them using the fitness function, and afterward we select only the best $\mu$ individuals from a $(\mu+\lambda)$ population and return to determine if some model has reached a good match with the observed spectrum.

Here we have employed a modified version of ES that includes some changes to the canonical version [15]. We create $\mu=11$ parent individuals and $\lambda=22$ children individuals; we use a $(\mu+\lambda)$ scheme for selection, discrete recombination, and traditional mutation, but we also include a new way to create offspring with the average operator and a dynamical mutation [16] for strategy parameter vectors based on a simple, but effective and easy to understand, multiplication by constant factors. The pseudocode of the ES algorithm for this problem is the following:

1) Create $\mu$ parent vectors $\vec{x}_{i} ; i=1, \ldots, \mu$ where each vector contains 12 parameters $\vec{x}_{i}=$ $\left[a_{1, i}, a_{2, i}, a_{3, i}, m_{1, i}, m_{2, i}, m_{3, i}, r_{1, i}, r_{2, i}, r_{3, i}, c_{1, i}\right.$, $\left.c_{2, i}, c_{3, i}\right]$, and their corresponding strategy parameter vectors $\vec{\sigma}_{i} ; i=1, \ldots, \mu$ where $\vec{\sigma}_{i}=\left[\sigma_{1}, \sigma_{2}, \ldots, \sigma_{12}\right]$. Each parameter is chosen through a random process and satisfying the constraints of the problem.

2) For each vector $\vec{x}_{i}$ produce a simulated galaxy spectrum $\vec{g}_{i}$

3) For each $\vec{g}_{i}$ compute the fitness function $f\left(\vec{g}_{i}\right)=\sum_{\lambda=3800}^{\lambda=8000}\left|o_{\lambda}-g_{i, \lambda}\right| w_{\lambda}$

4) If some model $\vec{g}_{i}$ fits good the observed spectrum terminate, otherwise continue next step

5) Create new $\lambda$ individuals in the following way:

- Create $10 \%$ of $\lambda$ population using discrete recombination, from two parents $\vec{x}_{a}$ and $\vec{x}_{b}$, chosen randomly, we create a new children $\vec{x}_{k}^{\prime}$ :

$$
\begin{aligned}
& x_{k, j}^{\prime}=x_{a, j} \text { or } x_{b, j} ; j=1,2, \ldots, 12 \\
& \sigma_{k, j}^{\prime}=\sigma_{a, j} \text { or } x_{b, j} ; j=1,2, \ldots, 12
\end{aligned}
$$

- Create $10 \%$ of $\lambda$ population using average operator, from two parents $\vec{x}_{a}$ and $\vec{x}_{b}$, chosen randomly:

$$
\begin{aligned}
& \vec{x}_{k}^{\prime}=w \vec{x}_{a}+(1-w) \vec{x}_{b} \\
& \vec{\sigma}_{k}^{\prime}=w \vec{\sigma}_{a}+(1-w) \vec{x}_{b}
\end{aligned}
$$

where $w$ is a uniform random number between 0 and 1 .

- Create $80 \%$ of $\lambda$ population using mutation: 


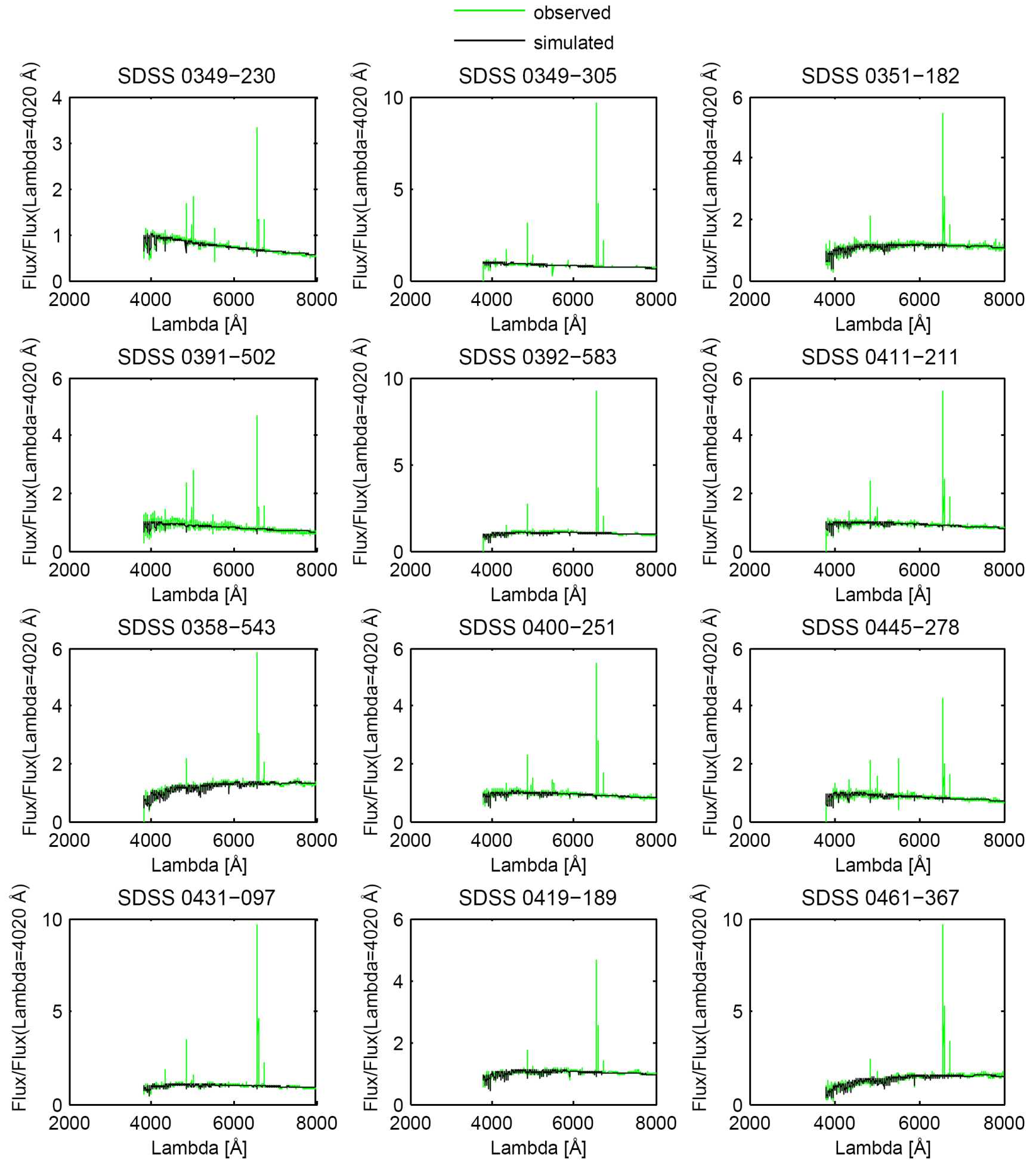

Fig. 3. Fitting for a sample of spectra from SDSS DR2, showing observed spectra in thick lines and models in tick lines. Spectra are normalized in the region $\lambda=4020 \AA$ 


$$
\vec{x}_{k}^{\prime}=\vec{x}_{a}+N\left(0, \vec{\sigma}_{a}\right)
$$

6) For each vector $\vec{x}_{k}^{\prime}$ produce a simulated galaxy spectrum $\vec{g}_{k}^{\prime} ; k=1, \ldots, \lambda$

7) For each $\vec{g}_{k}^{\prime}$ compute the fitness function $f\left(\vec{g}_{k}^{\prime}\right)=\sum_{\lambda=3800}^{\lambda=8000}\left|o_{\lambda}-g_{k, \lambda}^{\prime}\right| w_{\lambda}$

8) Merge $\mu$ and $\lambda$ populations to obtain a $(\mu+\lambda)$ population

9) Sort the merged population by fitness function

10) Select the best $\mu$ individuals from the sorted population

11) Mutate strategy parameter vectors:

$$
\vec{\sigma}_{i}^{\prime}=\left\{\begin{array}{l}
0.3 \vec{\sigma}_{i} \text { if } \vec{x}_{i} \text { is a child } \\
3.3 \vec{\sigma}_{i} \text { if } \vec{x}_{i} \text { is a parent }
\end{array}\right.
$$

where these values have been selected experimentally. In this manner when we have successful populations (parent individuals) then we can explore farther regions from the current point, trying to improve the current best fitness; while when we have unsuccessful populations (children individuals) we explore nearer regions to current point with the same purpose.

12) If some model $\vec{g}_{i}$ fits the observed spectrum well or the maximum number of generations is reached terminate, otherwise return to 5

\section{RESUlts}

In order to evaluate the efficiency of the evolutionary method ES in this problem we have produced results for a sample of 100 spectra taken from SDSS DR2 and used in [12].

The fitting of one galaxy spectrum takes on average 1 minute on a PC with a PIV $3 \mathrm{GHz}$ processor and 512 MB of RAM, using MatLab ${ }^{\circledR}$. Computational time can be improved if we employ a compiled language such $\mathrm{C}$, $\mathrm{C}++$ or FORTRAN rather than an interpreted one. In [7] authors comment that their program based on Simulated Annealing take 4 minutes to fit an spectrum on a $2 \mathrm{Ghz}$ Linux workstation using a $\mathrm{C}$ compiler.

The fitness function 2 is not normalized, so it describes the fitness of each individual by measuring the direct difference of its corresponding model with respect to the observed spectrum. We say a model $\vec{g} *$ matches perfectly the observed spectrum when $f(\vec{g} *)=0$, but since we are matching models with observational data, we do not expect to reach the real minimum. Rather, we are interested in obtaining good approximations to the observed spectra.

After a set of experiments we determined that a value of 60 for the fitness function is good enough and the model can be acceptable, a smaller value for this threshold would lead to a better fit, albeit at an increase in computation time.

We illustrate the fitting with a sample of 12 examples, presented in Figure 3 that shows 12 spectra from SDSS
TABLE II

RESULTS FOR A SET OF 100 GALAXY SPECTRA FROM SDSS DR2

\begin{tabular}{|c|c|c|c|}
\hline \hline $\begin{array}{c}\text { Function Evaluations } \\
\text { (average) }\end{array}$ & $\begin{array}{c}f(\vec{g}) \\
\text { (average) }\end{array}$ & $\begin{array}{c}\text { Standard } \\
\text { deviation }\end{array}$ & $\begin{array}{c}\text { Success } \\
(\%)\end{array}$ \\
\hline \hline 5005 & 26.576 & 12.65 & 85 \\
\hline \hline
\end{tabular}

DR2 fitted by models approximated by ES. We can observe that, as stated before, emission lines are not fitted but the general distribution of absorption lines and the curve form are approximated very closely. A better fit is observed around the $4020 \AA$ region because of the normalization, and the less fitted part is at the end of the spectra because the models from the library are known not to be as accurate in this range of wavelengths. Also, as settled in the previous paragraph, we are not expecting to match perfectly the observed spectra, but to find good descriptions of the absorption lines.

In Table II we present the summarized results for a sample of 100 galaxy spectra. From this table we can observe that we have reached the goal threshold of 60 for fitness function in $85 \%$ of the analyses. In this work we established experimentally a limit of 5005 function evaluations (for a total of 455 generations) for ES, and a limit of 1 for the fitness function to terminate. We can see from the table that no one fitting was able to reach the lower limit for the fitness function, and all of them had to spend the maximum number of allowable function evaluations. The average fitness function value is 26.576, which means a good fitting for the great majority of the spectra, with a minimum value of 8.46 and a maximum value of 59.82, having a standard deviation for the sample of 12.65, which means we do not have a standard or uniform fitting, rather the fitting is related with the particular details of the observed spectrum.

We have mentioned that a similar problem has been solved using other methods like Simulated Annealing [7] and $\chi^{2}$ minimization [6], nevertheless we do not present comparisons with these methods because they fit a different set of galaxy spectra, and then a direct comparison is not suitable.

\section{Conclusions and Future Work}

In this paper we have presented a novel application of ES in astronomy, showing the versatility and good behavior of ES in difficult problems. Spectral fitting is a complex non-trivial problem that normally has been solved with other methods such as $\chi^{2}$ minimization or Simulated Annealing, but that are normally expensive in terms of computational time. With ES the spectral fitting was successful; the algorithm was able to find good models in almost all the spectra from the sample, with a relatively low computational cost, taking on average 1 minute to fit one spectrum, which is a very good time if we take into account that are dealing with real data. In this manner ES have proved to be a very well suited method for this problem.

For future work we can employ a parallelized version of our ES algorithm, to reduce computational time with larger 
samples of data. In addition, a better fit can be obtained if we use model libraries that take into account the gas component, and with better resolution for all the wavelength range. Also we can obtain improvements if we include other effects such as velocity dispersion in the models.

\section{ACKNOWLEDGEMENTS}

This work was partially supported by CONACYT, Mexico, under grants C02-45258/A-1 and 144198.

\section{REFERENCES}

[1] I. Rechenberg, Evolutionsstrategie: Optimierung technischer Systeme nach Prinzipien der biologischen Evolution. Stuttgart: FrommannHolzboog, 1973.

[2] D. Ashlock, Evolutionary Computation for Modeling and Optimization. New York: Springer, 2005.

[3] L. Giraud-Moreau and P. Lafon, "A comparison of evolutionary algorithms for mechanical design components," Eng. Opt., vol. 34, no. 1, pp. 307-322, 2002.

[4] S. Vazquez-Montiel, J. Sanchez-Escobar, and O. Fuentes, "Obtaining the phase of an interferogram by use of an evolution strategy: Part i." Applied Optics, vol. 41, no. 17, pp. 3448-3452, 2002.

[5] G. Fernandez, S. Muñoz, R. A. Sanchez, and W. W. Mayol, "Simultaneous stabilization using evolutionary strategies," International Journal of Control, vol. 68, no. 6, pp. 1417-1435, 1997.

[6] M. J. Wolf, N. Drory, K. Gebhardt, and G. J. Hill, "Ages and metallicities of extragalactic globular clusters from spectral and photometric fits of stellar population synthesis models," The Astrophysical Journal, vol. 655 , no. 1, pp. 179-211, 2007.

[7] R. C. Fernandes, A. Mateus, L. Sodré, G. Stasin'ska, and J. M. Gomes, "Semi-empirical analysis of sloan digital sky survey galaxies - i. spectral synthesis method," Monthly Notices of the Royal Astronomical Society, vol. 358, no. 2, pp. 363-378, 2005.

[8] J. C. Gomez, L. Athanassoula, O. Fuentes, and A. Bosma, "Using evolution strategies to find a dynamical model for the m81 triplet," in Lecture Notes in Artificial Intelligence, Oct. 2004, pp. 404-410.

[9] H. Karttunen, P. Kroger, H. Oja, M. Poutanen, and K. Donner, Fundamental Astronomy. Berlin, Heidelberg: Springer-Verlag, 2000.

[10] A. Bressan, C. Chiosi, and F. Fagotto, "pectrophotometric evolution of elliptical galaxies. 1: Ultraviolet excess and color-magnitude-redshift relations," The Astrophysical Journal Supplement Series, vol. 94, no. 1, pp. 63-115, 1994.

[11] K. Abazajian et al., "The second data release of the sloan digital sky survey," The Astronomical Journal, vol. 128, no. 1, pp. 502-512, 2004.

[12] M. Bello, "Estudio de la evolución temporal del triplete de ca ii," Master's thesis, INAOE, Puebla, Mexico, 2007.

[13] D. J. Schlegel, D. P. Finkbeiner, and M. Davis, "Maps of dust infrared emission for use in estimation of reddening and cosmic microwave background radiation foregrounds," The Astrophysical Journal, vol. 500, no. 1 , pp. 525-, 1998.

[14] J. A. Cardelli, G. C. Clayton, and J. S. Mathis, "The relationship between infrared, optical, and ultraviolet extinction," The Astrophysical Journal, vol. 345, no. 1, pp. 245-256, 1989.

[15] T. Back, U. Hammel, and H. P. Schwefel, "Evolutionary computation: Comments on the history and current state," IEEE Trans. Evol. Comput., vol. 1, no. 1, pp. 3-17, 1997.

[16] H. Beyer, "Toward a theory of evolution strategies: Self-adaptation," Evolutionary Computation, vol. 3, no. 3, pp. 311-347, 1996. 